\title{
Thoraco-Lumbar Junction Disc Herniation and Tight Filum: A Unique Combination?
}

\author{
Erwin M. J. Cornips'1, Emile A. M. Beuls², Biene W. Weber ${ }^{3}$, Johannes S. H. Vles ${ }^{3}$ \\ ${ }^{1}$ Department of Neurosurgery, Maastricht University Medical Center, Maastricht, The Netherlands \\ ${ }^{2}$ Department of Forensic Medicine and Pathology, University Hospital Antwerp, Antwerp, Belgium \\ ${ }^{3}$ Department of Child Neurology, Maastricht University Medical Center, Maastricht, The Netherlands \\ Email: e.cornips@mumc.nl
}

Received 22 April 2014; revised 20 May 2014; accepted 13 June 2014

Copyright (C) 2014 by authors and Scientific Research Publishing Inc.

This work is licensed under the Creative Commons Attribution International License (CC BY).

http://creativecommons.org/licenses/by/4.0/

\section{Abstract}

Purpose: The incidence of both symptomatic thoraco-lumbar junction disc herniation (TLJDH) and tight filum (TF) may be underestimated. Both conditions have a complex clinical presentation that may involve the distal spinal cord, conus medullaris, and/or cauda equina, including upper and/or lower motor neuron impairment, sensory impairment, urological and sexual dysfunction. The coexistence of both conditions has not been previously reported and may be a diagnostic and therapeutic challenge. Methods: We report three teenage girls, a 24-year-old woman, and two middleaged women who were diagnosed with both conditions and treated at our institution. Results: Disc herniation level was T11-T12 in 2, T12-L1 in 3, and L1-L2 in one. All patients had a fatty filum (n = 5) and/or a low-lying CM (at or above L1-L2 in 2, at or below L2-L3 in 4), and were treated with filum sectioning first. All patients noted marked improvement of preoperative complaints including back pain $(n=5)$, leg pain and fatigue $(n=4)$, urological complaints $(n=4)$, and toe gait $(n=1)$. One 16-year-old girl successfully underwent a thoracoscopic microdiscectomy for persisting pain at the thoraco-lumbar junction two years after filum sectioning. Conclusions: Thoraco-lumbar junction disc herniation and tight filum both act on the distal spinal cord close to the transition to the cauda equina. Both conditions may coincide and may even act synergistically, the disc herniation acting as a fulcrum, aggravating the deleterious effect of the tethering force (and vice versa). This might explain why both conditions combined may present at a younger age. We suggest filum sectioning as the primary treatment option in all patients, however, more cases and a longer follow-up are needed to better understand their unique combination and interaction. Nevertheless, when confronted with a symptomatic TLJDH especially in young patients we advise to rule out a coinciding TF by careful consideration of all clinical, radiological, and urological data.

\section{Keywords}

Clinical Presentation, Disc Herniation, Tethered Cord, Therapy, Thoraco-Lumbar Junction, Tight 


\section{Filum}

\section{Introduction}

The thoraco-lumbar junction (TLJ) in its anatomical definition comprises the T12 and L1 vertebrae, however, considering the biomechanical weakness of T11 having free edged ribs, as well as individual variations in the location of the caudal end of the spinal cord, Tokuhashi [1] proposed the TLJ in its clinical definition comprises four vertebrae from T11 to L2. In the present paper, we adhere to the latter definition, according to which the TLJ includes three motion segments: T11-T12, T12-L1, and L1-L2. Of note, because of the variable number of ribs and lumbar vertebrae, the only way to ensure correct vertebral level counting is to use full-spine magnetic resonance (MR) scans in each case [2] [3].

Disc herniations at T11-T12 are true thoracic disc herniations (TDHs) as they are situated in the thoracic spine and impinge on the caudal spinal cord. Disc herniations at T12-L1 and L1-L2 are neither true thoracic nor true lumbar disc herniations, as they are situated at the transition between caudal spinal cord, epiconus (myelomeres L4-S2), conus (myelomeres S3-S5), and cauda equina. The filum terminale (FT) starts at the caudal end of the conus medullaris (CM), situated between the lower third of the T12 vertebral body and the middle third of the L2 vertebral body in the vast majority of patients [3]. A tight filum (TF) is defined as a short, thick, and/or nonelastic filum, with or without a low-lying CM [4]. Both TDHs and TF have a widely variable clinical presentation that may mimic a broad range of other diseases. As such, both entities are difficult to diagnose, and their true incidence may be underestimated even to date [1] [2] [4]-[14].

Literature on thoraco-lumbar junction disc herniations (TLJDHs) and their clinical presentation is scant [1] [7] [9] [11]. They may cause lower back pain and ischialgia [11], upper and/or lower motor neuron disease [1] [7] including a so-called compressive lumbar myelopathy [9], micturition problems [1] [7], and sphincter problems [1] [7]. Moreover, the fact that TDHs may present with merely pain (either axial, band-like, or irradiating to the legs) without symptoms or signs of myelopathy, though reported as early as 1956 [5], is not widely acclaimed in recent literature [15].

Literature on TDHs and TLJDHs in the pediatric population is very scant [6] [8] [16]-[18], whereas literature on symptomatic tight filum, the so-called tight filum syndrome (TFS), is quite abundant [3] [4] [19]. The combination of TF and TLJDH, to the best of our knowledge, has not been previously reported, let alone in a pediatric case. In the present paper, we report three teenage girls, a 24-year-old woman, and two middle-aged women who were diagnosed with both entities, and treated with filum sectioning first. We elaborate on their clinical presentation, imaging findings, pathophysiology, surgical strategy, and postoperative outcome.

\section{Case Material (Table 1)}

We present six patients (all female) in order of ascending age (range 12 - 58 years) diagnosed with a TLJDH and a fatty filum and/or a low-lying CM (Table 1). All patients had failed conservative therapy and were offered filum sectioning through an interlaminar approach. At each follow-up patient-related neurological status was classified as overall worsened, improved, or unchanged from preoperative baseline with regard to pain, motor, and/ or urinary dysfunction.

\subsection{Case 1 (Figure 1)}

A 12-year-old girl presented with a one year history of aching pain at the TLJ irradiating to her flank and legs, fatigue in her legs after a short walk, urinary urge, incomplete bladder emptying, and nocturnal incontinence. We observed a normal gait, vivid leg reflexes, and marked tenderness at the TLJ. MR imaging demonstrated discopathies at the TLJ, a small TDH at T10-T11 and larger broad-based TDH at T12-L1, a low-lying CM at L3-L4, and a LF (Figure 1). Urodynamic testing revealed a neurogenic bladder. We believed most findings except maybe for tenderness at the TLJ could be explained by a tethered cord and decided to cut the filum. Three months postoperatively, she reported no more urinary urge or incontinence, no more fatigue in her legs, and markedly less pain at the TLJ no longer interfering with daily activities. She has been doing fine for almost two years now. 
Table 1. Case characteristics.

\begin{tabular}{cccccc}
\hline Case \# & Age & Sex & TLJDH level & CM level & Filum aspect \\
\hline $\mathbf{1}$ & 12 & F & T12-L1 & L3-L4 & mid L1 \\
$\mathbf{2}$ & 14 & F & T12-L1 & L2-L3 & LF \\
$\mathbf{3}$ & 15 & F & T11-T12 & L1-L2 & N \\
$\mathbf{4}$ & 24 & F & L1-L2 & L5-S1 & LF \\
$\mathbf{5}$ & 53 & F & T11-T12 & low L3 & LF \\
\hline
\end{tabular}

CM, conus medullaris; F, female; L, lumbar; LF, lipomatous filum; N, normal; T, thoracic; TLJDH, thoraco-lumbar junction disc herniation; S, sacral.
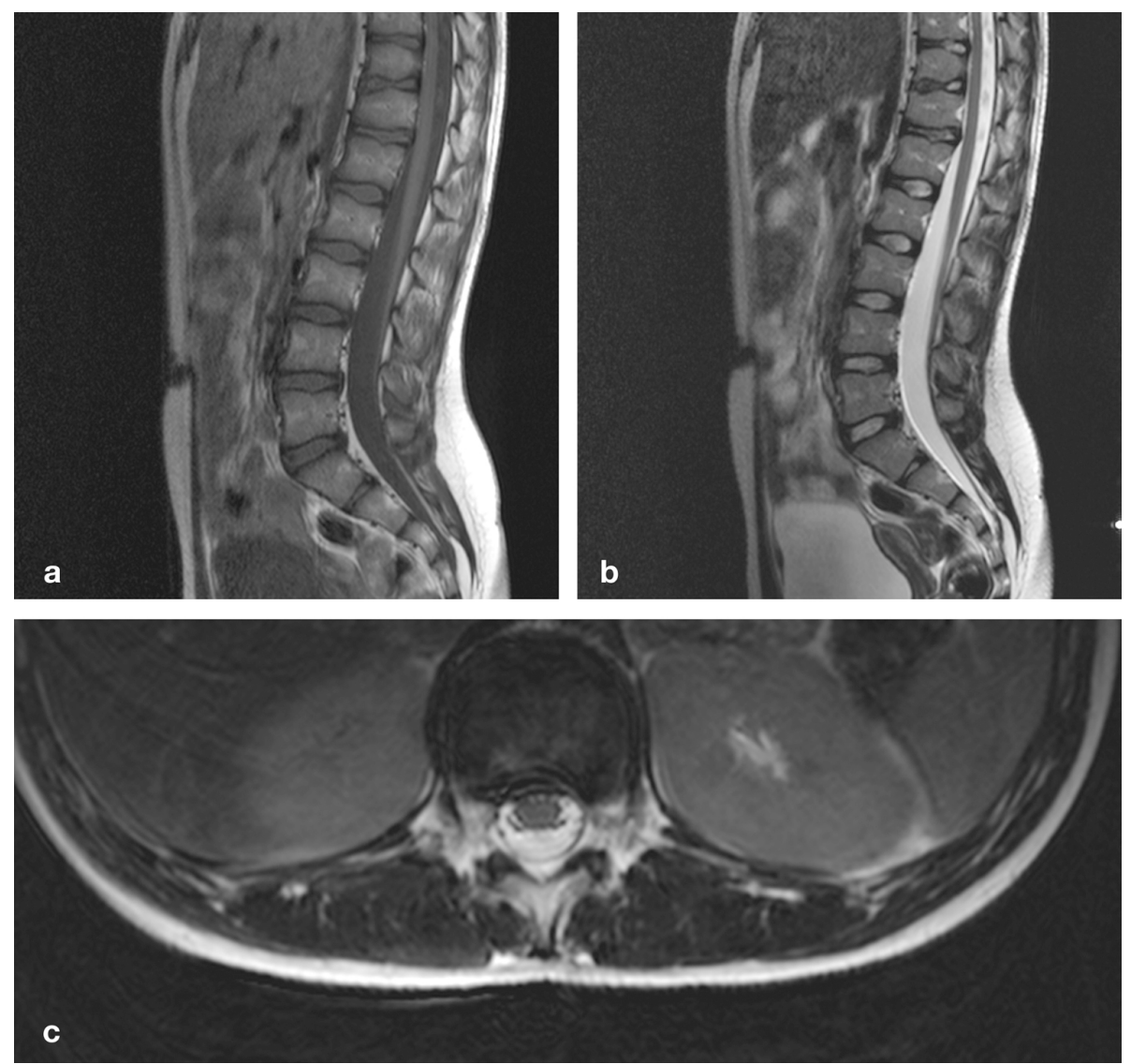

Figure 1. Sagittal T1 (a) and T2-weighted (b) MR images demonstrate a tapered spinal cord at L3-L4 and multiple discopathies at the TLJ. Axial T2-weighted MR image highlights a broad-based disc herniation at T12-L1, impinging on the spinal cord (c). T, thoracic; L, lumbar; TLJ, thoraco-lumbar junction.

\subsection{Case 2 (Figure 2)}

A 14-year-old girl and talented gymnast suddenly experienced aching pain at the TLJ irradiating to the left flank, and a completely blocked anteflexion. She was unable to sit or walk more than 15 minutes, let alone to perform gymnastics, and remained severely incapacitated despite pain killers and physiotherapy. When she came to our attention one year later, we noticed tense paraspinal muscles, marked tenderness over the T12-L1 motion segment, a completely blocked anteflexion, and painful redression. Micturition was normal. MR imaging demonstrated a broad-based TDH at T12-L1, impinging on the CM less than $2 \mathrm{~cm}$ above its termination (Figure 2). 

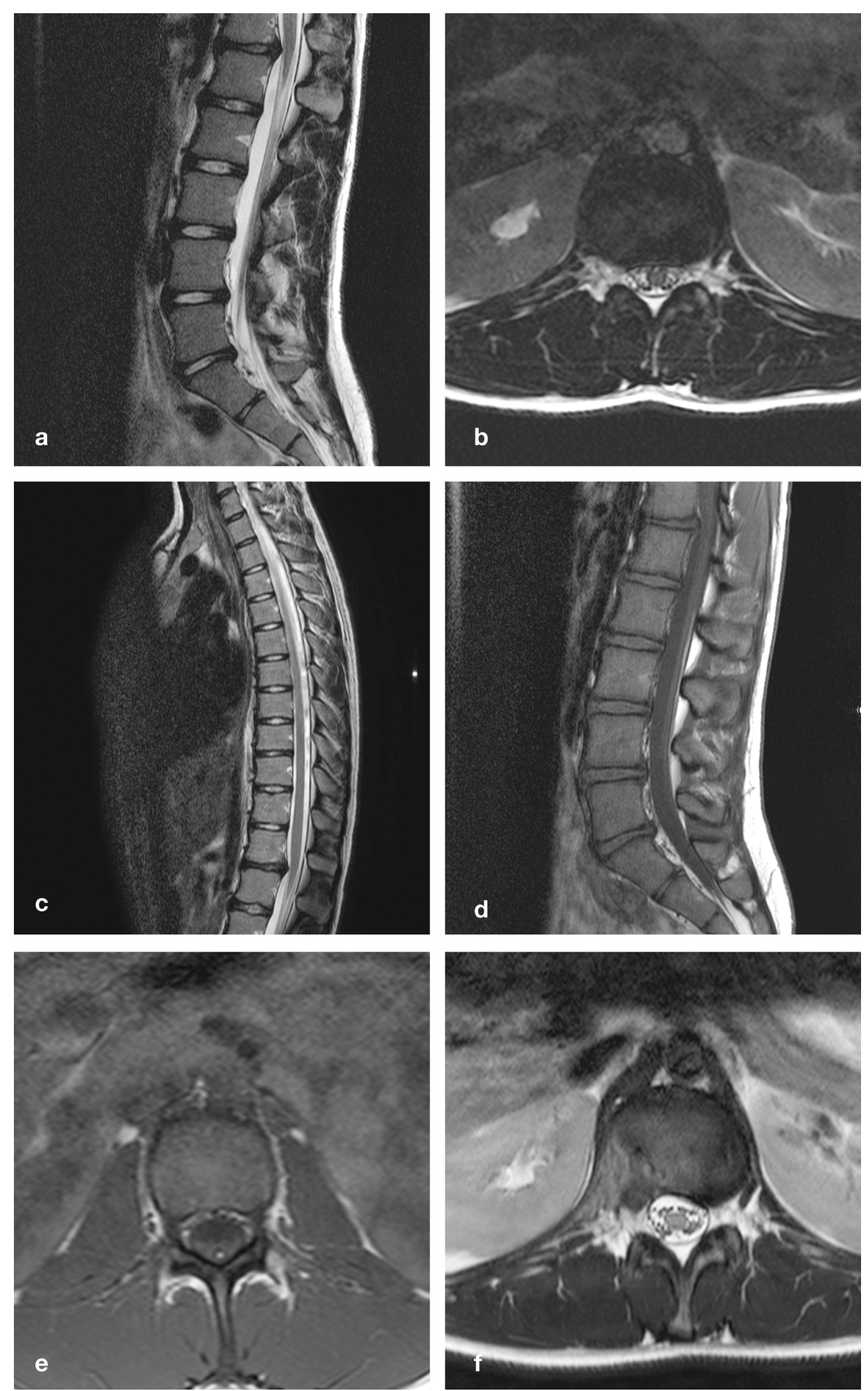

Figure 2. Sagittal (a) and axial (b) T2-weighted MR images demonstrate degenerative discopathy and a broad-based DH at T12-L1, impinging on the conus less than $2 \mathrm{~cm}$ above its termination. Additional sagittal T2-weighted MR image (c) rules out other DHs, whereas sagittal (d) and axial (e) T1-weighted MR images clearly demonstrate a lipomatous filum at the L2 and L3 vertebral level. Postoperative axial T2-weigthed MR image (f) demonstrates adequate decompression. DH, disc herniation; T, thoracic; L, lumbar. 
As spontaneous and provoked pain were consistently pointing to the T12-L1 motion segment, we considered a thoracoscopic microdiscectomy (TMD), but were reluctant to do so because of her young age, the absence of any neurological deficit, and the importance of this motion segment. Taking another look at the MR images, we noticed a slightly tapered aspect of the CM ending halfway L1, and more importantly a LF at L2-L3 (Figure 2). Although the clinical presentation suggested a symptomatic TDH rather than a tethered cord, we hypothesized the LF might be pulling the CM over the TDH, the latter acting as a fulcrum, aggravating the conflict between CM and TDH. Therefore we decided to cut the filum, hoping to reduce pain to an acceptable level, or at least to buy time until she was full-grown. Soon after surgery, she reported markedly less pain and demonstrated greatly improved anteflexion. At one year, she had resumed most social and school activities, but was unable to sport because of persisting pain at the TLJ. At two years, she finally had a TMD, and experienced immediate, complete pain relief. She has been doing fine for well over a year after her second operation.

\subsection{Case 3 (Figure 3)}

A 15-year-old girl was referred because of toe walking, which had always been present but was clearly progressive over the past few months. Besides toe walking, we observed an inability to stand on the heels, discrete dystrophy of lower leg and foot musculature, mild weakness of tibialis anterior muscles, vivid leg reflexes, bilateral Babinski sign, and tenderness over the T11-T12 motion segment. Micturition was normal. MR imaging demonstrated discopathy and a flat, broad-based, right mediolateral TDH at T11-T12. The conus-cauda transition was at L2-L3 with normal appearing filum (Figure 3). We decided to cut the filum, and although it did not appear thickened nor very tight intraoperatively, her toe gait improved and eventually disappeared in subsequent months.

\subsection{Case 4 (Figure 4)}

A 24-year-old woman presented with a nine month history of low back pain, non-radicular pain in the right leg, and diminished sensation in the left leg. The pain was continuous with sudden exacerbations during which she had to lay down despite diazepam, pregabaline, and morphine. By the end of the day, she tended to limp, and suffered involuntary muscle contractions in her legs as soon as she lay down. She also reported dysuria, pollakisuria, and urge incontinence. We noticed tenderness over the lumbar spine, markedly reduced anteflexion, normal appearing feet, vivid knee jerks, and hypesthesia over the anterior aspect of the left leg. She had postmictional residus up to 300 milliliters. MR imaging demonstrated kyphotic angulation and a broad-based TLJDH at L1-L2 impinging on the conus-cauda transition, as well as a LF extending from L2 to S1 (Figure 4). A few weeks after filum sectioning, she reported markedly reduced pain and demonstrated greatly improved
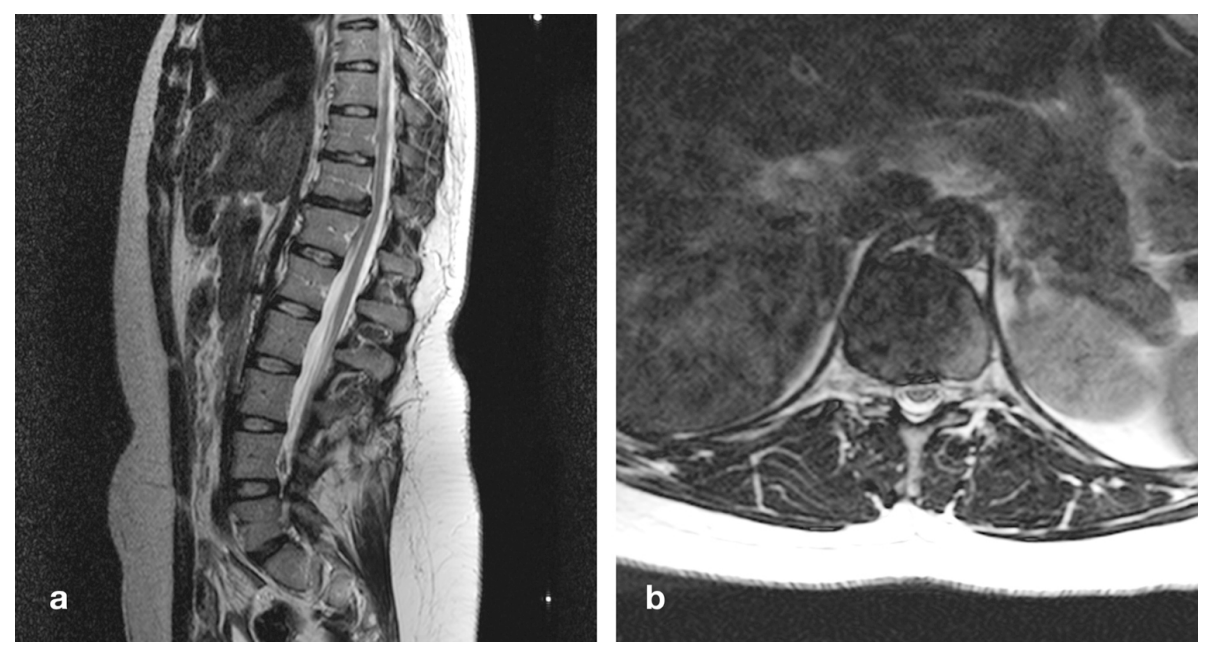

Figure 3. Sagittal (a) and axial (b) T2-weighted MR images after filum sectioning through an L4-L5 interlaminar approach. The conus-cauda transition was at L2-L3 (not shown). Note degenerative discopathy (a) and a flat, broad-based disc herniation at T11-T12 (a, b). T, thoracic; L, lumbar. 

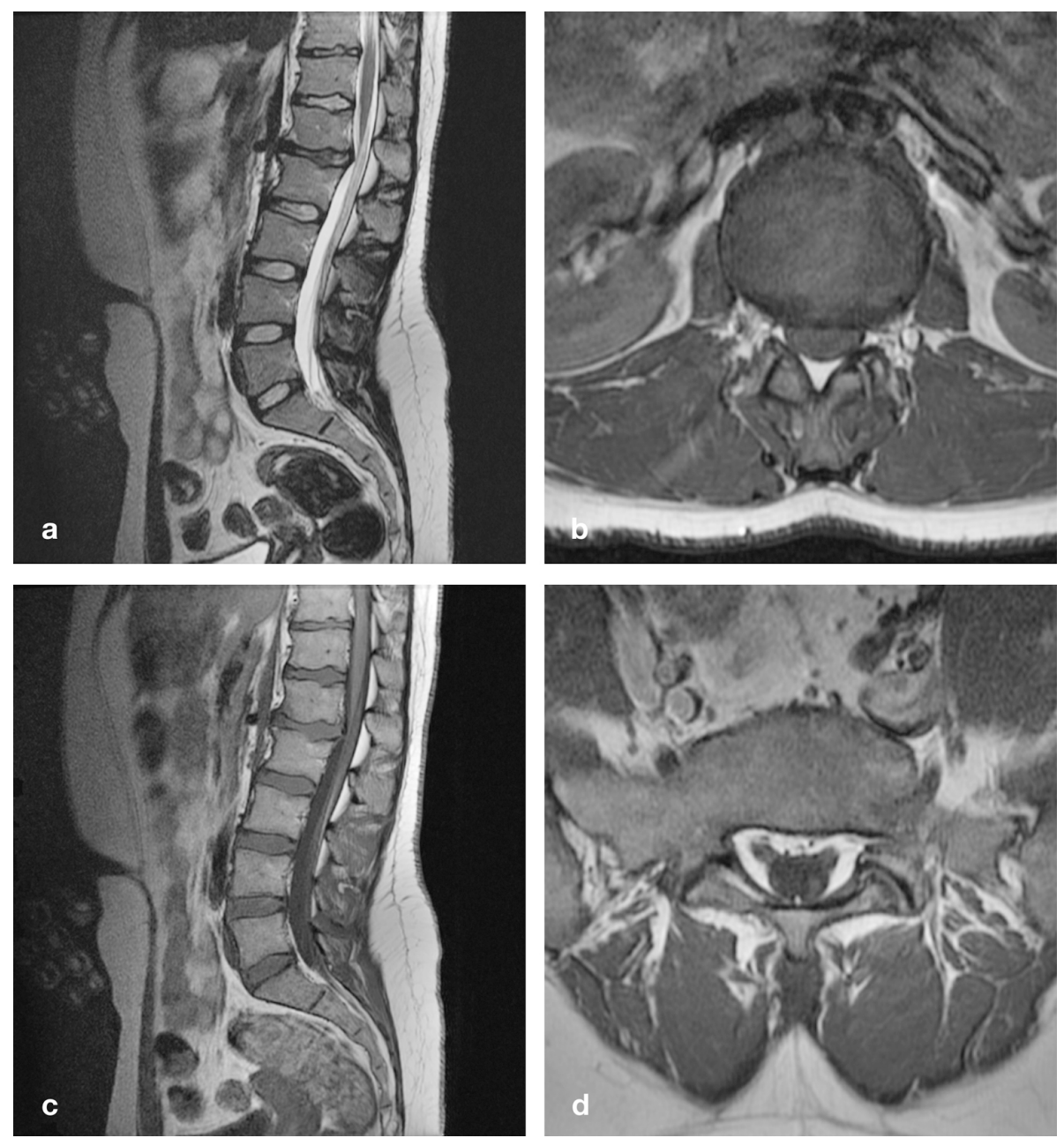

Figure 4. Sagittal T2 (a) and axial T1-weighted (b) MR images demonstrate kyphotic angulation and a broad-based DH at L1-L2 impinging on the conus-cauda transition. Sagittal (c) and axial (d) T1-weighted MR images demonstrate a lipomatous filum extending from L2 to S1. $\mathrm{DH}$, disc herniation; L, lumbar; S, sacral.

anteflexion without using any painkiller. Three months postoperatively, she reported minimal back pain even after work, normal sensation in the left leg, and a slight tendency to limp when very tired. All urinary complaints had disappeared.

\subsection{Case 5 (Figure 5)}

A 53-year-old woman presented with continuous, non-radicular pain and dysesthesia in her right leg, progressively worsening over the past two years, and more recently to a lesser extent in her left leg, gluteal and sacral area. By the end of the day she could hardly walk. She also reported urinary urge, incomplete bladder emptying, and sporadic urinary incontinence. We observed a small scar in the upper gluteal fold where a dimple had been surgically removed 30 years ago, a subcutaneous lipoma immediately above, vivid leg reflexes, decreased sensation in the right S2-S5 dermatomes, and marked tenderness over the T11-T12 motion segment. Plain X-ray images demonstrated discopathies at L1-L2 and L4-L5, and an open arch and sacralization at L5. MR imaging demonstrated a left paramedian TDH at T11-T12 with marked deformation of the spinal cord, a CM at L5-S1, and a LF at S1-S2 (Figure 5). Despite tenderness over the T11-T12 segment, we believed her clinical presentation pointed to a tethered cord, the TDH merely playing a secondary yet possibly aggravating role. A few weeks after filum sectioning, she reported markedly reduced pain, less urinary urge, and more complete bladder emptying. She resumed her full-time secretarial job despite intermittent pain at the TLJ irradiating over the upper 

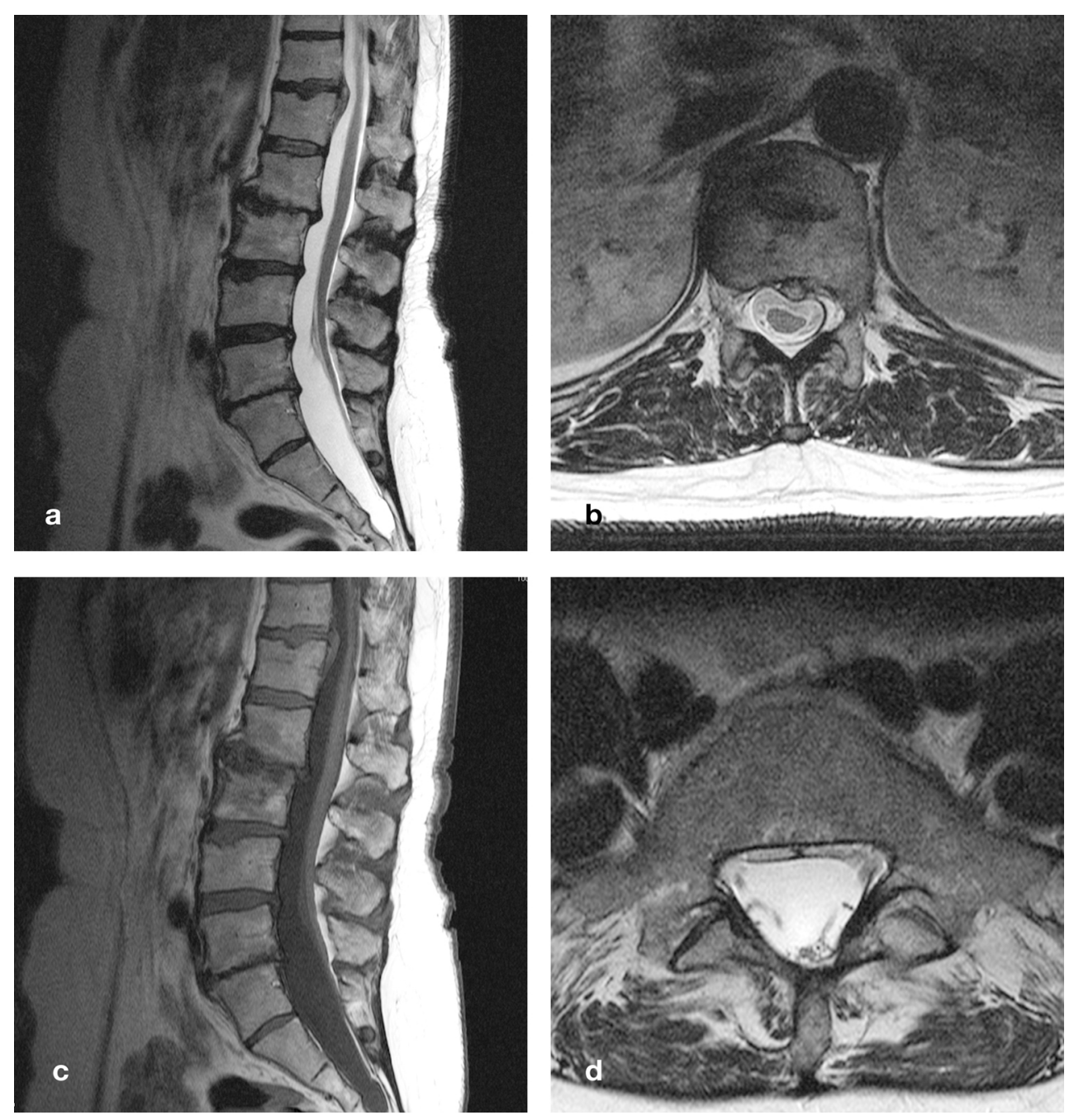

Figure 5. Sagittal (a) and axial (b) T2-weighted MR images demonstrate degenerative discopathy at L1-L2 and L4-L5, a left paramedian DH at T11-T12, and a low-lying conus at L5-S1. The spinal cord is clearly displaced and deformed even though (due to the supine scanning position) there appears to be no physical contact with the DH. Additional sagittal T1-weighted (c) and axial T2weighted (d) MR images demonstrate a thick, lipomatous filum at S1-S2. DH, disc herniation; T, thoracic; L, lumbar; S, sacral.

abdomen likely caused by the TDH. One year postoperatively, all pain had disappeared, while MR imaging demonstrated regression of the TDH.

\subsection{Case 6 (Figure 6)}

A 58-year-old woman presented with an eight year history of progressive low back pain and leg pain with acute exacerbations. She could walk less than 100 meters, was unable to lie down with her legs fully stretched, and also reported incomplete bladder emptying. We noticed an asymmetrical gluteal fold, weakness of the right tibialis anterior and extensor hallucis longus muscles, vivid leg reflexes, an indifferent right plantar response, hypesthesia in the right S1 (foot) and S2-S5 (perineum) dermatomes, and marked tenderness over the TLJ and lumbar spine. MR imaging demonstrated a right paramedian TDH at T12-L1 with deformation of the spinal cord, a CM at the lower border of L3, and a LF at L5 (Figure 6). Urodynamic testing was normal. A few days after filum sectioning, she was able to lie down with her legs fully stretched for the first time in many years. Six weeks postoperatively, she was able to walk a kilometer in a more upright posture, which further improved with physiotherapy the following months. 

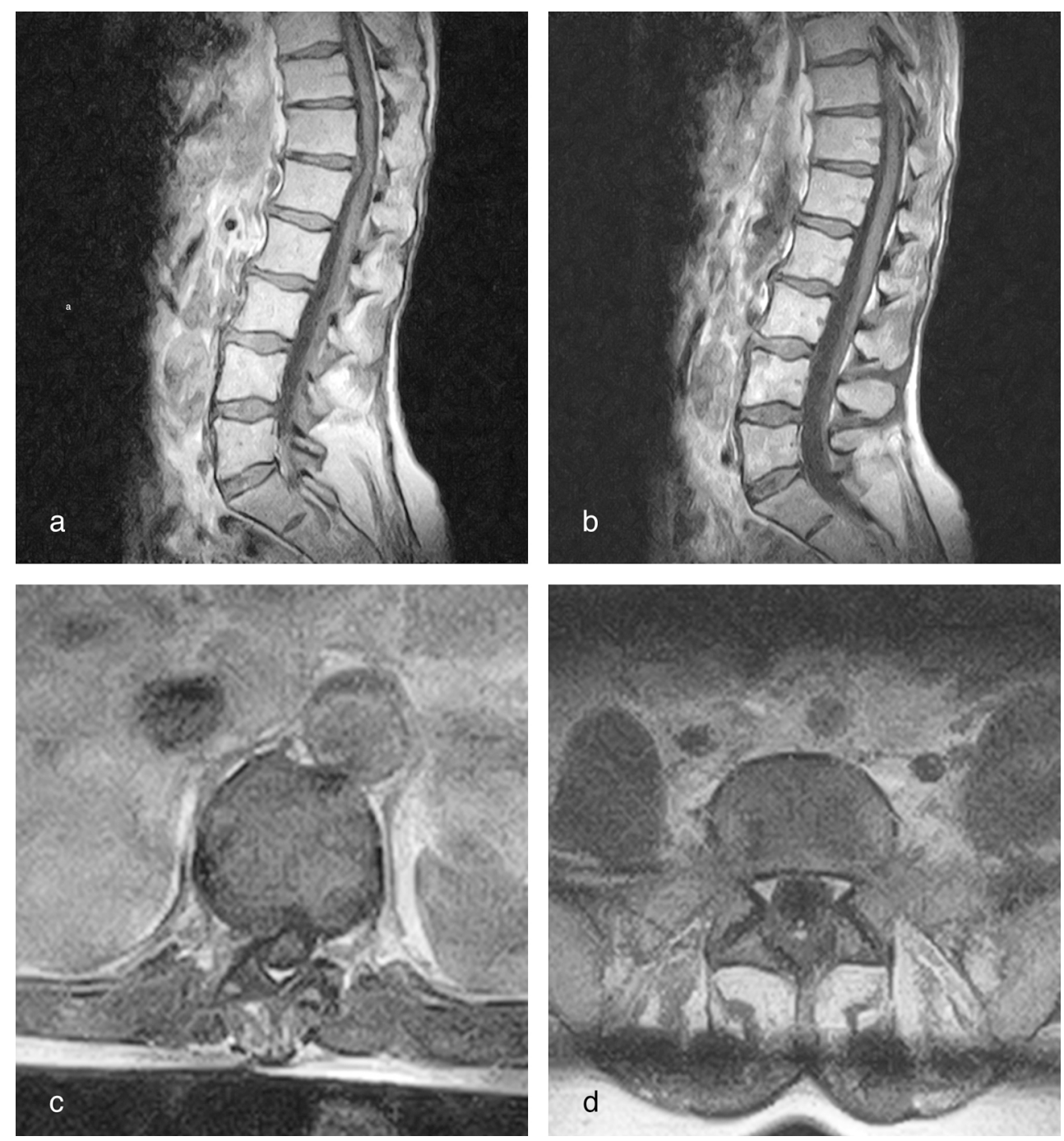

Figure 6. Midsagittal (a), slightly parasagittal (b) and axial (c) T1-weighted MR images demonstrate a right paramedian DH at T12-L1 with marked deformation of the spinal cord, a conus-cauda transition at the lower border of L3, and a lipomatous filum clearly visible on axial T1-weighted MR image at the L5 level (d). DH, disc herniation; T, thoracic; L, lumbar.

\section{Discussion}

\subsection{Incidence and Clinical Presentation of TLJDHs}

In the adult population, the incidence of symptomatic TDHs has been estimated at 1/1.000.000 [10] [12] [15] but may actually be higher considering a prevalence of $37 \%$ on MR imaging in asymptomatic individuals [20]. The incidence of symptomatic TLJDHs is essentially unknown as these have rarely been analyzed as a separate clinical entity [1] [7] [9] [15] [21] [22]. Functioning as a tertiary referral center, our surgical series encompasses 357 single or multi-level TMDs performed between October 2000 and April 2013. In this series, age ranges from 16 to 83 years, and involved level from T2-T3 to T12-L1, including 35 (9.8\%) TMDs at T10-T11, 52 (14.2\%) at T11-T12, and 9 (2.5\%) at T12-L1. Therefore, one quarter (26.5\%) of all symptomatic TDHs were situated at the TLJ.

In the pediatric population, there are merely case reports of symptomatic TDHs [6] [16]-[18], including one 16-year-old girl with a T10-T11 disc herniation at the upper limit of the TLJ [8]. These TDHs (especially when calcified and/or associated with Scheuermann's disease) may be related to a growth disturbance rather than to disc degeneration [12] [23]. They may be underdiagnosed because they are not included in the differential diag- 
nosis. Alternatively, they may be underreported because validated treatment options are lacking. Other TDHs may develop in the second decade only to become symptomatic many years later [2].

In anatomical terms, the L4-S2 myelomeres (epiconus) are usually located from the T11-T12 intervertebral disc over the T12 vertebral body, the S3-S5 myelomeres (conus) from the T12-L1 intervertebral disc over the L1 vertebral body, and the caudal end of the spinal cord between midcorporeal level L1 and midcorporeal level L2 [1]. However, individual variations are considerable, and even the most frequent location of the caudal end of the spinal cord at the L1-L2 intervertebral disc is observed in only 30\% of individuals [1] [3]. Thus, depending on the exact level of both disc herniation and caudal end of the spinal cord, the clinical presentation of TLJDHs may be one of distal spinal cord, CM, or cauda equina compression [1].

In clinical practice, TLJDHs are often misdiagnosed as lower lumbar disc disease [1] [11]. In 2003, Kleopa ea [9] published an interesting paper "Compressive lumbar myelopathy presenting as segmental motor neuron disease". These authors reported four patients with a T11-T12 disc herniation suffering a myelopathy with predominantly lower motor neuron symptoms and signs, caused by lower motor neuron loss at the corresponding spinal cord level, a condition which they termed compressive lumbar myelopathy (CLM). The most common initial symptom was slowly progressive asymmetric weakness in the legs, often presenting as foot drop, muscle waisting, and walking difficulties. All patients suffered fasciculations and cramps in the affected muscles. Interestingly, only one patient suffered low back pain early in the course of his disease, as opposed to five patients (all except case 3) in the present series. Moreover, not a single patient reported sensory loss or bowel/bladder dysfunction early in the course of his disease, as opposed to four patients (case 1, 4-6) in the present series. However, all patients eventually developed burning and tingling sensations in their feet, and one patient developed band-like abdominal pain [9].

Contrary to the observations of Kleopa ea [9] and illustrating the clinical variability of TLJDHs, Tokuhashi ea [1] observed spastic paraplegia caused by compression of the upper motor neuron with T10-T11 as well as T11-T12 disc herniations. According to these authors, such disc herniations should be considered upper motor neuron disorders, while T12-L1 disc herniations should be considered lower motor neuron disorders, and L1-L2 disc herniations should be considered combined disorders of cauda equina and radiculopathy. Moreover, there would be no neurologic findings conclusive for involvement at the T12-L1 level. Sanderson et al. [22] postulate L1-L2 and L2-L3 disc herniations may cause an ill-defined poli-radiculopathy because the spinal canal is much narrower at these levels and/or because of significant cross-innervation between spinal nerves in this region. Iwasaki et al. [24] postulate L1-L2 and L2-L3 disc herniations present with urinary disturbance significantly more often than their lower lumbar counterparts because the spinal canal is much narrower at these levels facilitating compression of the $\mathrm{CM}$ and/or multiple cauda equina nerves.

\subsection{Incidence and Clinical Presentation of TFS}

The true incidence of TFS in children is not known, however, the condition may be underdiagnosed because of its clinical variability [4]. The number of adults in whom a tethered cord syndrome (TCS) is diagnosed continues to grow as a result of better imaging and increased clinical awareness [13], however, the condition may be underdiagnosed as well because they experience a more gradual and insidious onset of symptoms and may never seek medical attention [13]. Moreover, because low-back pain and degenerative disc disease are very common, diagnosis is often misattributed to these entities, and the true incidence of adult TCS remains unknown [13].

The clinical presentation of TFS may be a combination of symptoms and signs in four categories: cutaneous, orthopedic, neurologic, and urologic/sexual [4] [19]. Adults and adolescents may present with variable neurological deficits, including any combination of upper and/or lower motor neuron symptoms and/or sensory impairment. As such, they may experience sensory deficits, muscle weakness, bladder and bowel dysfunction, skeletal and foot deformities, as well as pain which often is the most prominent symptom [13]. In some patients TFS may mimic lumbar disc disease [14], whereas in others it may cause pyramidal signs including hyperreflexia and Babinski signs [13]. In the present series, case 1 suffered pain and urological dysfunction (urologic), case 2 merely suffered pain, case 3 demonstrated toe walking and discrete dystrophy (neurologic), case 4 suffered sensory deficits (neurologic) and urological dysfunction (urologic), case 5 demonstrated a gluteal dimple (cutaneous) and suffered sensory deficits (neurologic) and urological dysfunction (urologic), and case 6 demonstrated an asymmetrical gluteal fold (cutaneous) and suffered sensory-motor deficits (neurologic) and urological dysfunction (urologic). Thus, we observed one, two, or even three positive symptom categories that may point to a 
TFS [4] [19] in all but one patient (case 2). On the other hand, hyperreflexia (all except case 2) may point to a TFS as well as a TDH with cord compression above the level of the CM.

\subsection{TF and TLJDHs Coinciding}

\subsubsection{Diagnostic Considerations}

To the best of our knowledge, the combination of TF and TLJDH has not been previously reported. We report 6 cases, all female, an observation for which we have no explanation at present. Although TF and TLJDH may coincide, as we have demonstrated, the combination may be missed as clinicians may be familiar with merely one condition, or may not be looking for another diagnosis once an initial diagnosis has been made. Indeed, a LF and normally positioned CM (as in case 2) or even a low-lying, tapered CM may be overlooked just as easily as a TLJDH at the upper margin of a sagittal MR image of the lumbosacral spine. Moreover, the clinical presentation of both conditions may involve the distal spinal cord, CM, or cauda equina, and include upper and/or lower motor neuron impairment, sensory impairment, urological and sexual dysfunction [1] [2] [4] [19]. This similarity makes it very difficult to differentiate both conditions, even though some findings may point to one condition in particular, for example spontaneous axial pain aggravated by palpation of the involved spinous process in case of a symptomatic TLJDH.

Case 1 and 2 presented with axial pain at the TLJ as the primary complaint, most likely pointing towards the T12-L1 disc herniation. On the other hand, case 3 merely had palpatory tenderness at the TLJ, case 4 merely had low back pain, case 5 had gluteal and sacral pain as well as palpatory tenderness at the TLJ, and case 6 had low back pain as well as palpatory tenderness at the TLJ and lumbar spine. Hence, case 3 (no spontaneous pain) and case 4-6 (low back pain) may have been less suggestive for a symptomatic TLJDH as far as their pain was concerned, however, weakness observed in the L5 musculature unilaterally (case 6) or bilaterally (case 3) might have been explained by a CLM [9] just as well as a TCS. Of note, merely one in four patients in the Kleopa (CLM) series [9] as opposed to all patients in the Tokuhashi (TLJDH) series [1] suffered low back pain early in the course of their disease.

Case 4 presented with unilateral lower limb hypesthesia, and case 5 and 6 presented with sacral hypesthesia, as opposed to none of the patients in the Kleopa (CLM) series [9], and all patients in the Tokuhashi (TLJDH) series [1]. Moreover, case 1 and 4-6 in the present series presented with urinary problems, as opposed to none of the patients in the Kleopa (CLM) series [9], and all patients affected by a T10-T11, T11-T12, or T12-L1 disc herniation, but none of the patients affected by an L1-L2 disc herniation in the Tokuhashi (TLJDH) series [1]. However, as sensory and urological symptoms and signs may be caused by a TCS as well [4] [19], they are not very helpful in differentiating TFS and symptomatic TLJDH.

\subsubsection{Pathophysiologic Considerations}

In 1981, Yamada [25] postulated that TCS is a stretch-induced functional disorder of the lumbosacral spinal cord due to excessive tension. Repetitive flexion and extension of the spine may cause unremitting traction on a tethered cord [13], producing local spinal cord ischemia and neuronal damage with worsening vascular and metabolic dysfunction over time [25]-[27]. Whereas the pathophysiology of TCS in general and TFS in particular have been extensively studied, little is known about the pathophysiology of TDHs [2] [12] [28] and TLJDHs [1]. We do know however the disc herniation causes compression and the filum causes stretching of the distal spinal cord and CM, the latter resulting in mitochondrial dysfunction [25]-[27]. We also know the absence of denticulate ligaments and the prominent pial fenestrations make the CM particularly vulnerable [29]. In case a TF and TLJDH coincide, both conditions will interact with the distal spinal cord and CM causing a complex pattern of symptoms and signs. We hypothesize a TF may aggravate the conflict between a TLJDH and the distal spinal cord or CM (and vice versa), resulting in an earlier (case 1-4 were less than 25 years old) and/or a more aggressive (case 4 and 6) clinical course. The TLJDH may act as a compressive force and as a fulcrum for the stretched distal spinal cord and CM, aggravating the deleterious effect of the tethering force (and vice versa) (Figure 7).

In 1982, Pang and Wilberger [30] published a paper on adult TCS, focusing on individuals who were normal in childhood and developed symptoms in adulthood, as well as individuals with static neurological deficits and/or skeletal deformities in childhood who remained well until the onset of new, progressive neurological deficits in adulthood. They postulated that the degree of traction on the CM determines the age of symptomatic 


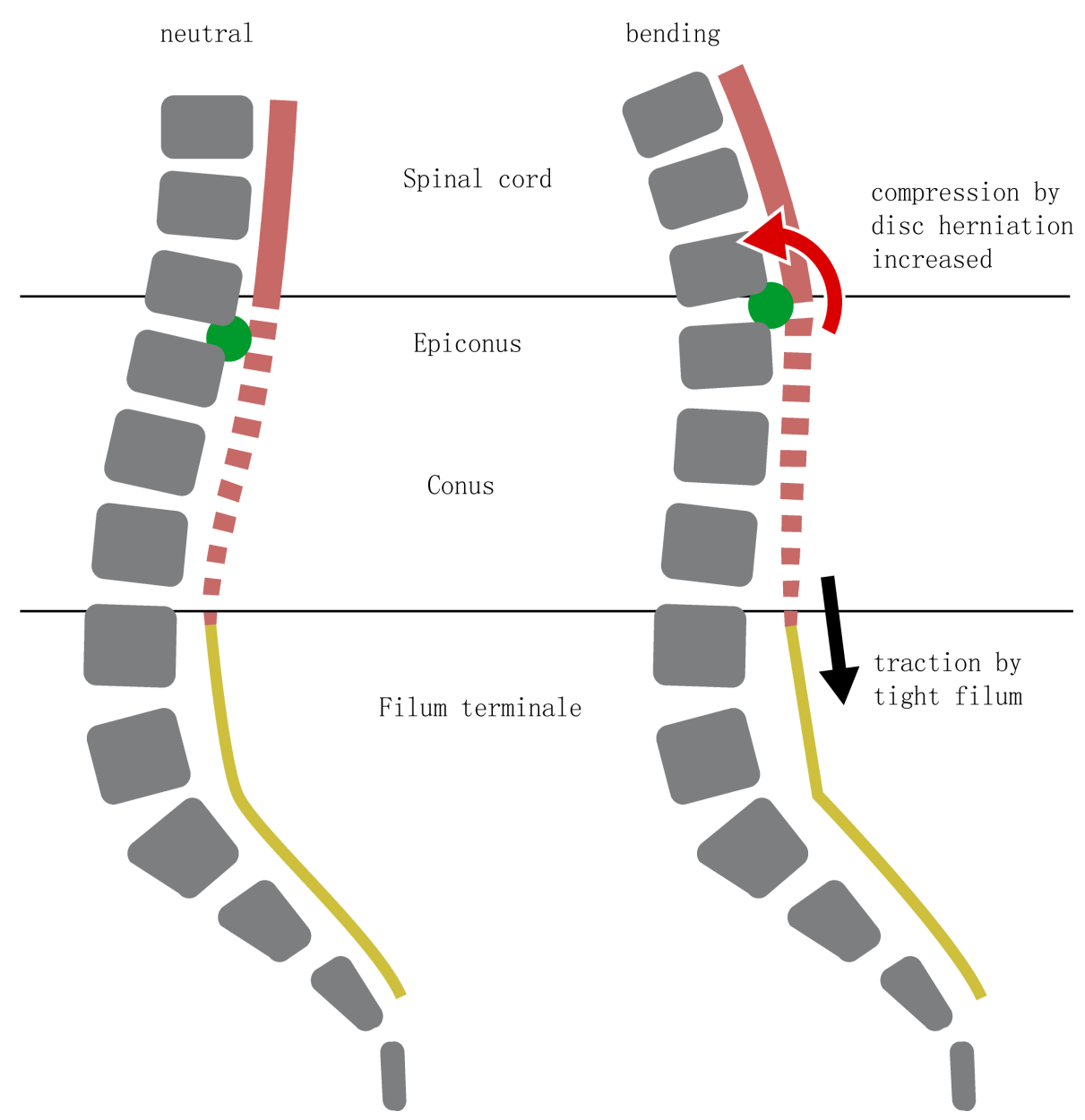

Figure 7. Postulated pathophysiological mechanism in case a TLJDH and TF coincide. Both spinal cord compression caused by the DH (green dot) and spinal cord traction (black arrow) caused by the TF (yellow line) increase with forward bending (red arrow). The DH may act as a fulcrum, aggravating the deleterious effect of the TF on an already compromised spinal cord. $\mathrm{DH}$, disc herniation; TF, tight filum; TLJDH, thoraco-lumbar junction disc herniation.

onset of a TCS, and identified aggravating factors that may upset the tenuous balance between abnormal tension and optimal cellular function later in life, leading to an acute onset of symptoms. These were either a direct blow to the back or a fall on the buttocks, momentary stretching of a presumably tight CM, or narrowing of the spinal canal and crowding of the intraspinal contents as caused by disc bulging, disc herniation, and/or degenerative spinal stenosis. Indeed, any disc herniation impinging on the ventral aspect of the spinal canal will push the already tightened spinal cord backward and accentuate the stretch. Clearly, our observations are in line with this theory. Moreover, we believe several factors may act synergistically (Figure 8), which might explain some cases presenting at very young age (case 1-3). Case 2 for example was a young girl presenting with a T12-L1 disc herniation, a LF, and a passion for gymnastics promoting repetitive trauma of her already compromised CM.

\subsubsection{Surgical Considerations and Outcome}

Both TF and TLJDH may cause unremitting pain and/or progressive neurological deficit unresponsive to conservative measures. Both conditions combined may severely incapacitate the patient and necessitate surgical intervention. The surgeon then has to decide whether to cut the filum (untethering) or to resect the disc herniation (cord decompression). In experienced hands, filum sectioning is a safe, straightforward procedure that does not impair spinal stability if performed through an interlaminar approach [4]. On the other hand, TMD although well established is a relatively more complex, technically demanding procedure, and even more so at the TLJ, where the diaphragm may be in the way [2] [15] [31] [32]. Moreover, because TLJ motion segments are more mobile 


\section{SPINAL CORD / CM COMPROMISE}

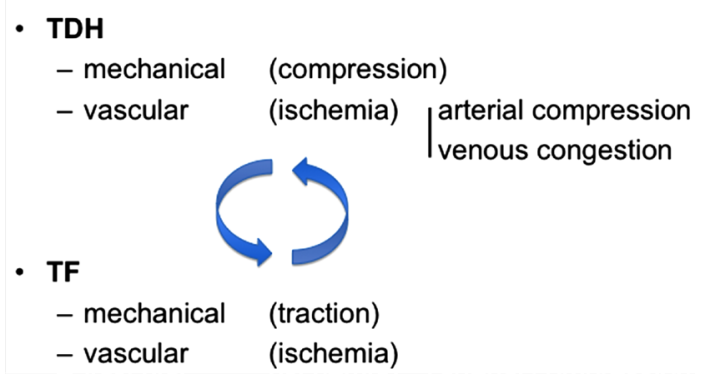

Figure 8. Postulated pathophysiological mechanism in case a TLJDH and TF coincide. Both entities may cause mechanical and vascular spinal cord compromise, reinforcing each others deleterious effects (blue arrows). TF, tight filum; TDH, thoracic disc herniation.

and bear more weight than any other thoracic level, it is important to preserve stability especially in not fullgrown individuals. Therefore, as long as there is no hyperintens spinal cord signal (myelomalacia) at the level of the TLJDH on T2-weighted MR images (none of our patients), it seems reasonable to perform filum sectioning first, and observe the clinical evolution thereafter. Using this strategy, we noted marked clinical improvement in all patients. Nevertheless, those who presented with pain (all except case 3) reported some persisting pain in the legs, at the TLJ, and/or at the upper abdomen, most likely due to the TLJDH. Interestingly, the pain gradually disappeared as the disc herniation regressed in one patient (case 5). On the other end of the spectrum, pain persisted despite maximal conservative measures only to disappear after resection of the disc herniation in a 16year-old girl (case 2), the only one who had a TMD in this series.

In conclusion, we believe that case 1, 2, 5, and 6 were likely symptomatic from a TTC as well as a TLJDH, while the disc herniation may have been merely an aggravating factor or even a coincidental finding, given the observed prevalence in asymptomatic individuals [20], in case 3 and 4. Clearly, more cases and a longer followup are needed to better delineate and understand the unique combination of both conditions. Nevertheless, when confronted with a symptomatic TLJDH especially in young patients we advise to rule out a coinciding TFS by careful consideration of all clinical, radiological, and urological data.

\section{Conclusion}

Because of their variable clinical presentation, the incidence of TLJDH and TF may be underestimated even to date. To the best of our knowledge, the combination of both has not been previously reported, let alone in the pediatric population. However, as early as 1982 Pang and Wilberger [30] postulated that narrowing of the spinal canal (for example by a disc herniation) might be a possible mechanism leading to the acute onset of an adult TCS. We have demonstrated TLJDH and TF may coincide, and hypothesize they may even act synergistically, the disc herniation acting as a fulcrum, aggravating the deleterious effect of the tethering force (and vice versa). We suggest filum sectioning as the primary treatment option in all patients, however, more cases and a longer follow-up are needed to better understand the unique combination and interaction of both conditions. Nevertheless, when confronted with a symptomatic TLJDH especially in young patients, we advise to rule out a coinciding TF by careful consideration of all clinical, radiological, and urological data.

\section{References}

[1] Tokuhashi, Y., Matsuzaki, H., Uematsu, Y. and Oda, H. (2001) Symptoms of Thoracolumbar Junction Disc Herniation. Spine, 26, 512-518. http://dx.doi.org/10.1097/00007632-200111150-00021

[2] Cornips, E.M.J., Janssen, M.L.F. and Beuls, E.A.M. (2011) Thoracic Disc Herniation and Acute Myelopathy: Clinical Presentation, Neuroimaging Findings, Surgical Considerations and Outcome. Journal of Neurosurgery (Spine), 14, 520-528. http://dx.doi.org/10.3171/2010.12.SPINE10273

[3] Kesler, H., Dias, M.S. and Kalapos, P. (2007) Termination of the Normal Conus Medullaris in Children: A Whole- 
Spine Magnetic Resonance Imaging Study. Neurosurgical Focus, 23, E7. http://dx.doi.org/10.3171/FOC-07/08/E7

[4] Cornips, E.M.J., Vereijken, I.M., Beuls, E.A.M., Weber, J.W., Soudant, D.L., van Rhijn, L.W., Callewaert, P.R. and Vles, J.S.H. (2012) Clinical Characteristics and Surgical Outcome in 25 Cases of Childhood Tight Filum Syndrome. European Journal of Paediatric Neurology, 16, 103-117. http://dx.doi.org/10.1016/j.ejpn.2011.07.002

[5] Abbott, K.H. and Retter, R.H. (1956) Protrusions of Thoracic Intervertebral Disks. Neurology, 6, 1-10. http://dx.doi.org/10.1212/WNL.6.1.1

[6] Brennan, M., Perrin, J.C.S., Canady, A. and Wesolowski, D. (1987) Paraparesis in a Child with a Herniated Thoracic Disc. Archives of Physical Medicine and Rehabilitation, 68, 806-808.

[7] Demetriades, A.K., Naik, S. and Gunasekera, L. (2010) Conus Medullaris Syndrome form a Transdural Disc Herniation at the Thoracolumbar Juntion. Acta Neurochirurgica, 152, 1081-1082. http://dx.doi.org/10.1007/s00701-009-0586-9

[8] Giblin, E.A. and Hochheiser, G.M. (2008) Thoracic Disk Herniation Resulting in Acutely Progressing Paraplegia in a Pediatric Patient. Pediatric Emergency Care, 24, 550-553. http://dx.doi.org/10.1097/PEC.0b013e318180ff1d

[9] Kleopa, K.A., Zamba-Papanicolaou, E. and Kyriakides, T. (2003) Compressive Lumbar Myelopathy Presenting as Segmental Motor Neuron Disease. Muscle Nerve, 28, 69-73. http://dx.doi.org/10.1002/mus.10405

[10] Love, J.G. and Kiefer, E.J. (1950) Root Pain and Paraplegia Due to Protrusions of Thoracic Intervertebral Disks. Journal of Neurosurgery, 7, 62-69. http://dx.doi.org/10.3171/jns.1950.7.1.0062

[11] Lyu, R.K., Chang, H.S., Tang, L.M. and Chen, S.T. (1999) Thoracic Disc Herniation Mimicking Acute Lumbar Disc Disease. Spine, 24, 416-418. http://dx.doi.org/10.1097/00007632-199902150-00025

[12] Overvliet, G.M., Beuls, E.A.M., Ter Laak-Poort, M. and Cornips, E.M.J. (2009) Two Brothers with a Symptomatic Thoracic Disc Herniation at T11-T12: Clinical Report. Acta Neurochirurgica, 151, 393-396. http://dx.doi.org/10.1007/s00701-009-0244-2

[13] Rajpal, S., Tubbs, R.S., George, T., Oakes, W.J., Fuchs, H.E., Gadley, M.N. and Iskandar, B. (2007) Tethered Cord Due to Spina Bifida Occulta Presenting in Adulthood: A Tricenter Review of 61 Patients. Journal of Neurosurgery: Spine, 6, 210-215. http://dx.doi.org/10.3171/spi.2007.6.3.210

[14] Umur, A.S., Selcuki, M., Selcuki, D., Beduk, A. and Doganay, L. (2008) Adult Tethered Cord Syndrome Mimicking Lumbar Disc Disease. Child's Nervous System, 24, 841-844. http://dx.doi.org/10.1007/s00381-007-0539-7

[15] Anand, N. and Regan, J.J. (2002) Video-Assisted Thoracoscopic Surgery for Thoracic Disc Disease: Classification and Outcome Study of 100 Consecutive Cases with a 2-Year Minimum Follow-Up Period. Spine, 27, 871-879. http://dx.doi.org/10.1097/00007632-200204150-00018

[16] Bajard, X., Renault, F., Benharrats, T., Mary, P., Madi, F. and Vialle, R. (2010) Intervertebral Disc Calcification with Neurological Symptoms in Children: Report of Conservative Treatment in Two Cases. Child's Nervous System, 26, 973-978. http://dx.doi.org/10.1007/s00381-010-1117-y

[17] Nicolau, A., Diard, F., Darrigade, J.M., Dorcier, F. and Vital, J.M. (1985) Posterior Hernia of a Calcified Disk in Children. Apropos of 2 Cases. Journal de Radiologie, 66, 683-688.

[18] Wu, X.D., Chen, H.J., Yuan, W., Wang, X.W., Zhou, X.H., Li, H., Sheng, X.L. and Xu, S.M. (2010) Giant Calcified Thoracic Disc Herniation in a Child: A Case Report and Review of the Literature. Journal of Bone and Joint Surgery. American Volume, 92, 1992-1996. http://dx.doi.org/10.2106/JBJS.I.01652

[19] Fabiano, A.J., Khan, M.F., Rozzelle, C.J. and Li, V. (2009) Preoperative Predictors for Improvement after Surgical Untethering in Occult Tight Filum Terminale Syndrome. Pediatric Neurosurgery, 45, 256-261. http://dx.doi.org/10.1159/000228983

[20] Wood, K.B., Garvey, T.A., Gundry, C.R. and Heithoff, K.B. (1995) Magnetic Resonance Imaging of the Thoracic Spine. Evaluation of Asymptomatic Individuals. Journal of Bone and Joint Surgery. American Volume, 77, 1631-1638.

[21] Kim, D.S., Lee, J.K., Jang, J.W., Ko, B.S., Lee, J.H. and Kim, S.H. (2010) Clinical Features and Treatments of Upper Lumbar Disc Herniations. Journal of Korean Neurosurgical Society, 48, 119-124. http://dx.doi.org/10.3340/jkns.2010.48.2.119

[22] Sanderson, S.P., Houten, J., Errico, T., Forshaw, D., Bauman, J. and Cooper, P.R. (2004) The Unique Characteristics of “Upper” Lumbar Disc Herniations. Neurosurgery, 55, 385-389. http://dx.doi.org/10.1227/01.NEU.0000129548.14898.9B

[23] Kapetanos, G.A., Hantzidis, P.T., Anagnostidis, K.S. and Kirkos, J.M. (2006) Thoracic Cord Compression Caused by Disk Herniation in Scheuermann’s Disease. European Spine Journal, 15, 553-558. http://dx.doi.org/10.1007/s00586-005-0053-0

[24] Iwasaki, M., Akino, M., Hida, K., Yano, S., Aoyama, T., Saito, H. and Iwasaki, Y. (2011) Clinical and Radiographic Characteristics of Upper Lumbar Disc Herniation: Ten-Year Microsurgical Experience. Neurologia Medico-Chirurgica 
(Tokyo), 51, 423-426. http://dx.doi.org/10.2176/nmc.51.423

[25] Yamada, S., Zinke, D.E. and Sanders, D. (1981) Pathophysiology of “Tethered Cord Syndrome”. Journal of Neurosurgery, 54, 494-503. http://dx.doi.org/10.3171/jns.1981.54.4.0494

[26] Yamada, S., Iacono, R.P., Andrade, T., Mandybur, G. and Yamada, B.S. (1995) Pathophysiology of Tethered Cord Syndrome. Neurosurgery Clinics of North America, 6, 311-323.

[27] Yamada, S., Won, D.J., Pezeshkpour, G., Yamada, B.S., Yamada, S.M., Siddiqi, J., Zouros, A. and Colohan, A.R. (2007) Pathophysiology of Tethered Cord Syndrome and Similar Complex Disorders. Neurosurgical Focus, 23, 1-10. http://dx.doi.org/10.3171/FOC-07/08/E6

[28] McInerney, J. and Ball, P.A. (2000) The Pathophysiology of Thoracic Disc Disease. Neurosurgical Focus, 9, 1-8. http://dx.doi.org/10.3171/foc.2000.9.4.2

[29] Reina, M.A., De Leon Casasola, O., Villanueva, M.C., Lopez, A., Maches, F. and De Andres, J.A. (2004) Ultrastructural Findings in Human Spinal Pia Mater in Relation to Subarachnoid Anesthesia. Anesthesia \& Analgesia, 98, 1479-1485. http://dx.doi.org/10.1213/01.ANE.0000113240.09354.E9

[30] Pang, D. and Wilberger Jr., J.E. (1982) Tethered Cord Syndrome in Adults. Journal of Neurosurgery, 57, 32-47. http://dx.doi.org/10.3171/jns.1982.57.1.0032

[31] Regan, J.J., Mack, M.J. and Picetti III, G.D. (1995) A Technical Report on Video-Assisted Thoracoscopy in Thoracic Spinal Surgery. Preliminary Description. Spine, 20, 831-837. http://dx.doi.org/10.1097/00007632-199504000-00018

[32] Rosenthal, D., Rosenthal, R. and de Simone, A. (1994) Removal of a Protruded Thoracic Disc Using Microsurgical Endoscopy: A New Technique. Spine, 19, 1087-1091. http://dx.doi.org/10.1097/00007632-199405000-00018 
Scientific Research Publishing (SCIRP) is one of the largest Open Access journal publishers. It is currently publishing more than 200 open access, online, peer-reviewed journals covering a wide range of academic disciplines. SCIRP serves the worldwide academic communities and contributes to the progress and application of science with its publication.

Other selected journals from SCIRP are listed as below. Submit your manuscript to us via either submit@scirp.org or Online Submission Portal.
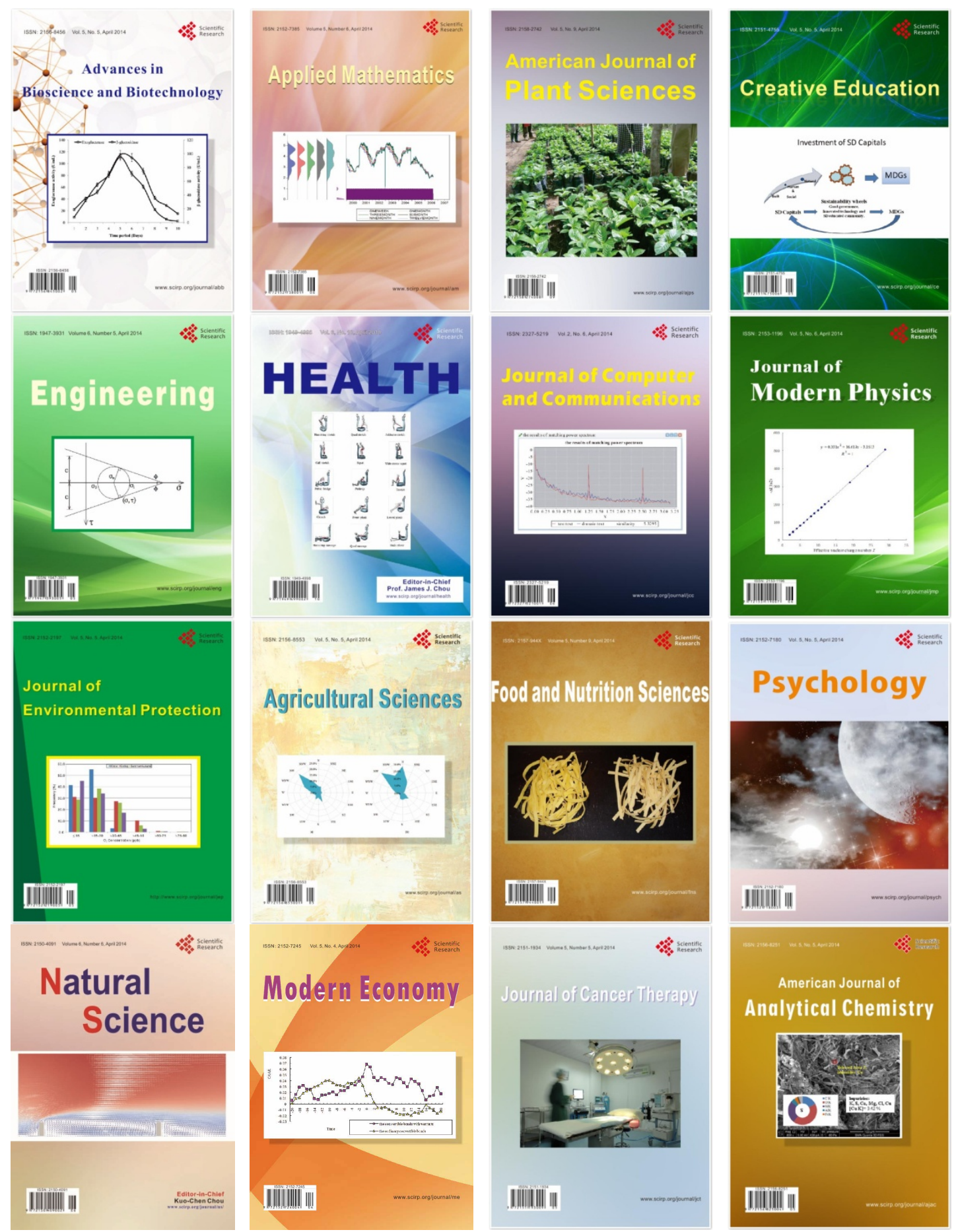